\title{
Aumento de Precisión en Localización Indoor basado en Redes Neuronales
}

\author{
Increased Accuracy in Indoor Location based on Neural Networks
}

\author{
Gerez Agustin", Goñi Oscar Enrique ${ }^{\# 1}$, Lucas Leiva ${ }^{\# 2}$ \\ ${ }^{\#}$ LabSET - INTIA, Fac. Cs. Exactas, Universidad Nacional del Centro de la Prov. de Buenos Aires \\ Pinto 399, (7000) Tandil, Pcia. De Buenos Aires, Argentina \\ ${ }^{1}$ oegoni@labset.exa.unicen.edu.ar \\ 21leiva@labset.exa.unicen.edu.ar \\ Recibido: 16/10/20; Aceptado: 27/11/20
}

\begin{abstract}
Resumen - La tecnología WiFi es ampliamente utilizada por un gran número de dispositivos, incluyendo aquellos que componen sistemas de Internet de las Cosas (IoT) y de Inteligencia Artificial (IA). En ambos contextos, el problema de localización ha sido objeto de investigación durante mucho tiempo. En algunos casos, las señales de radio utilizadas para transmitir información son además aprovechadas para realizar estimaciones de posición. Sin embargo, este enfoque se encuentra afectado por la constante fluctuación de la señal. Es posible que al momento de realizar una estimación de posición de un componente emisor, éste se encuentre influenciado por los obstáculos, el multitrayecto y la reflexión de la señal. Sin embargo, su uso mejora cuando se realiza localización espacial considerando diferentes referencias. De esta manera, es posible trazar activos dentro de un ambiente indoor. En este trabajo se analiza la relación de los algoritmos de estimación de distancia utilizando RSSI y triangulación, y se propone una solución basada en Redes Neuronales que combina los resultados de tres algoritmos de estimación de distancia con el fin de aumentar la precisión.
\end{abstract}

Palabra clave: localización, triangulación, distancia, redes neuronales

\begin{abstract}
The use of WiFi is widely used by a large number of devices, including those that make up the Internet of Things (IoT) and Artificial Intelligence (AI) systems. The location problem has been under investigation for a long time. In some cases, the radio signals used to transmit information are also used to make position estimates. However, its use is affected by the constant fluctuation of the signal. It is possible that when estimating the position of a component, it is influenced by obstacles, multipath and signal reflection. Its use improves when spatial localization is carried out, where assets can be traced within an indoor environment. In this work, the relationship of the distance estimation algorithms using RSSI and triangulation is analyzed, and a solution based on Neural Networks is proposed that combines the results of three distance estimation algorithms in order to increase precision.
\end{abstract}

Keywords: localization, triangulation, distance, ANN

\section{INTRODUCCIÓN}

La posición de un usuario o activo en su entorno puede resultar de un gran interés para numerosas aplicaciones, tal como el muestreo de información personalizada de la zona donde se encuentra el usuario, informar de zonas de especial interés por algunas peculiaridades como aglomeraciones o alertas meteorológicas. Este problema se ha resuelto en entornos a cielo abierto gracias al uso de GPS[1], GLONASS, Galileo u otros sistemas de localización de exteriores.

En los últimos años se ha dado gran auge a las tecnologías inalámbricas y como consecuencia a los servicios de localización. Su aplicación ha tenido una gran expansión tanto en interiores como en zonas urbanas, dando lugar a la aparición de una serie de servicios de comunicaciones que las utilizan debido a su sencillez y confiabilidad. De esta forma, las tecnologías de localización por ondas de radio ya funcionan con suficiente precisión en exteriores, motivo que hace lógico pensar que el siguiente paso es aplicar una tecnología similar dentro de edificios, tales como museos o centros comerciales[2]. En estos ambientes, este sistema puede ser utilizado para guiar al público hacia sitios de interés, como por ejemplo, una obra de arte en un museo, u ofrecer contenido acorde a la ubicación como la publicidad al pasar por una tienda en un centro comercial.

Si bien la localización en interiores (también llamada IPS, Indoor Position System) es un problema con algunas soluciones, estas carecen de la precisión necesaria para ciertas aplicaciones. El uso de GPS en ambientes interiores no es aplicable debido a la escasa penetración de las señales satelitales. Los equipos comerciales en este caso reportan directamente la información como no válida. Por esta razón, no existe un estándar que resuelva el posicionamiento en ambientes interiores. Existen ya varios productos comerciales que utilizan la comunicación inalámbrica entre varios dispositivos para determinar la ubicación de un móvil 
o activos pero en general poseen limitaciones en su precisión ya sean tecnológicas o por el enfoque de posicionamiento utilizado.

En el contexto de la localización en interiores, el problema puede ser resuelto a través del uso de tecnologías como las redes de área local inalámbrica (WLAN), también conocida como Wi-Fi, las redes de área personal inalámbrica (WPAN) que incluye identificación por radiofrecuencia (RFID), la banda ultra ancha (UWB) y Bluetooth de baja energía (BLE) [3]. Se debe considerar que, las señales inalámbricas más comunes poseen variación de intensidad en el nivel de señal debido a la presencia de obstáculos, o por las características del ambiente [4,5].

Respecto a los métodos de inferir la localización, éstos se clasifican en los basados en el rango y los de rango libre $[6,7]$. En el enfoque basado en el rango, los nodos deciden su posición teniendo en cuenta el cálculo de ángulo o distancia de algunos nodos de anclaje (nodos con posiciones conocidas) $[8,9]$. Dichas estimaciones pueden obtenerse a través de diversos procedimientos, por ejemplo, evaluando el tiempo de llegada (ToA) [10], evaluando la diferencia de tiempo de llegada (TDA) [11], estimando la dirección llegada (DoA) [12] o considerando el indicador de intensidad de señal de recepción (RSSI) [13].

En este trabajo se aborda un análisis comparativo de tres métodos (Zhu y Feng [14], Barai, Biswal y Sau [15], y Nasca y Teofil Cojocariu [16]) de localización en interiores enmarcados los enfoques de RSSI y triangulación. Con el objetivo de lograr un incremento en la precisión, este trabajo contribuye con una solución basada en una red neuronal artificial del tipo perceptrón simple que fusiona las salidas de los algoritmos e incrementa la precisión del cálculo de la distancia respecto a cada uno de los métodos seleccionados. Estas distancias son utilizadas luego para inferir la localización espacial. Para ello se utiliza el método de triangulación, realizando la linealización del sistema de ecuaciones cuadrático. Luego, se resuelve el sistema de ecuaciones lineal formado, ya sea usando eliminación Gaussiana o mediante otros métodos derivados del álgebra matricial.

El trabajo se organiza de la siguiente manera: en la sección II se describe y presenta el marco teórico, es decir, las principales características de los métodos seleccionados para la estimación de distancia y localización, la sección III presenta los resultados experimentales de la evaluación de cada uno de los enfoques y el desarrollo de la solución basada en el uso de una red neuronal. Finalmente, en la sección IV se presentan conclusiones y trabajos futuros.

\section{Marco teorico}

\section{A. Sistemas de localización}

En la actualidad, existen diversos sistemas que se utilizan para la localización ya sea de personas como de activos en interiores. Las tecnologías de RFID combinadas con estándares inalámbricos cómo ZigBee y Bluetooth, permiten el seguimiento de objetos en el plano sobre puntos discretos (o checkpoints) sin comprometer la movilidad de los mismos. La precisión de estos sistemas queda sujeto a la densidad de estos puntos.
Si bien los sistemas basados en ondas de radio dependen de una infraestructura tecnológica (por ejemplo, constelaciones de satélites o redes de acceso WiFi), es común encontrar zonas que ya dispongan de la misma. En aplicaciones como por ejemplo la navegación satelital, existen cuatro sistemas de funcionamiento que utilizan esta técnica de posicionamiento, siendo la implementación más conocida como el Sistema de Posicionamiento Global(GPS), de origen estadounidense. Los otros tres son Galileo(europeo), GLONASS(ruso) y BeiDou(chino).

Para que un receptor satelital pueda ser localizado, necesita recibir señales de al menos cuatro satélites. Cuanto mayor sea el número de satélites encontrados, más precisa será la estimación de la ubicación. Al recibir la posición actual del satélite, y midiendo el tiempo de envío de la señal, se utiliza el método conocido como trilateración para calcular la posición del receptor dentro del planeta.

Los sistemas de posicionamiento satelital han sido adoptados ampliamente debido a su integración nativa en los teléfonos móviles actuales. Es una tecnología confiable y de fácil acceso para el público general. Su principal inconveniente es que necesita una línea de visión directa entre el dispositivo receptor y al menos cuatro satélites, y hay situaciones donde este requisito no puede cumplirse, por ejemplo dentro de edificios, en túneles o en un yacimiento minero. Otra limitación importante es que su disponibilidad está ligada a entidades gubernamentales, motivo por el cual no es utilizada como fuente de información primaria en actividades militares. Considerando también que la precisión de estos sistemas suele ser variable, ya sea por condiciones atmosféricas, ambientales o gubernamentales, su precisión lo hace inviable para el posicionamiento en interiores.

Por otro lado, Wi-Fi es una tecnología inalámbrica que permite a usuarios conectarse inalámbricamente entre ellos a través de dispositivos conocidos como Access Point(AP) o punto de acceso para intercambio de información digital. Opera generalmente en las frecuencias de $2.4 \mathrm{GHz}$ y $5 \mathrm{GHz}$ y fue estandarizado por la IEEE como 802.11. Si bien las redes de $5 \mathrm{GHz}$ alcanzan velocidades mayores a las $2.4 \mathrm{Ghz}$, éstas son menos eficaces a la hora de traspasar obstáculos como paredes, muros y muebles, lo que resulta en un rango de menor cobertura. Su aplicación para la localización en interiores se logra mediante el uso de varios puntos de acceso anclados en posiciones conocidas.

El uso masivo de redes WiFi en edificios, escuelas o depósitos hacen que la localización en interiores sea posible utilizando la infraestructura ya existente. Sin embargo, la principal desventaja es que el protocolo 802.11 no fue diseñado con el objetivo de ser utilizado de esta manera: cada vez que se quiere realizar un escaneo para determinar la ubicación, se envían mensajes a todos los puntos de acceso Wi-Fi solicitando la información correspondiente, y esto impacta negativamente en el rendimiento de la red.

\section{B. Algoritmos de estimación de distancia}

Se propone el análisis de tres algoritmos de localización indoor, seleccionados por su bajo costo de implantación ya que no requieren de hardware o infraestructura adicional. Los algoritmos utilizan el método RSS para captar la señal 
de transmisión. Este método se basa en medir la atenuación de la señal recibida para inferir la localización del móvil y estimar la distancia que separa el móvil de cada Access Point (AP).

$$
P L(d B)=P L\left(d_{0}\right)-10 n-\log _{10}\left(\frac{d_{i}}{d_{0}}\right)
$$

El trabajo presentado por Zhu y Feng [14], propone una solución a la localización de interiores a partir de la fórmula de pérdida de ruta de camino libre, definida en la Ecuación 1 , y del concepto de atenuación. Mediante el uso del método de triangulación y el uso de matrices se determina la posición del individuo o activo. Una de las desventajas de este enfoque es que el objeto a localizar debe ubicarse donde al menos hay tres AP. En la Ecuación 1, $d_{0}$ representa la distancia de referencia cercana a la Tierra, $P L\left(d_{0}\right)$ es la intensidad de señal medida desde $d_{0}$, y $n$ es el factor de atenuación de la señal. Según el trabajo Zhu y Feng, el valor del factor varía entre 2 y 6 dependiendo del ambiente.

En el trabajo de Barai, Biswal y Sau [15], se realizan mediciones entre dos nodos determinando la relación entre el desvío estándar y la media de la intensidad de señal recibida (RSSI). A partir de esta relación, se determina la ecuación de distancia

$$
d_{A}=\frac{\left(-0.043 x^{5}-4.92 x^{4}-171.5 x^{3}-600.8 x^{2}+41.41 x-0.84\right)}{\left(x^{4}+250 x^{3}+14780 x^{2}-455.9 x+12.24\right)}
$$

Según Barai, Biswal y Sau[15], la relación se basa en que la desviación estándar más cercana a uno es más baja, y los valores RSSI están cercanos a la media para una distancia particular. Para la desviación estándar mayor a 3 los datos se incrementan y los valores de RSSI se extienden en un rango alto desde la media para una distancia particular [1]. Como resultado exitoso, los autores obtuvieron un nivel de error de $8.32 \%$

Finalmente, el trabajo de Nasca y Cojocariu [16], presenta una solución utilizando la fórmula de pérdida de ruta de espacio libre, teniendo en cuenta la potencia del transmisor y la frecuencia del sensor. Su solución es viable aunque se ve afectado por la latencia en las actualizaciones de los AP.

$$
d=10^{\frac{K-(P t x+\log F+\mid L L)}{20}}
$$

A partir de la fórmula de pérdida básica de transmisión en el espacio libre, se deduce la distancia d medida en metros según la Ecuación 3, donde $P t x[\mathrm{dBm}]$ es la potencia del transmisor, siendo $\mathrm{F}[\mathrm{MHz}]$ la frecuencia y $K$ es una constante que depende de las unidades de la distancia y frecuencia. Por último, $\mathrm{L}[\mathrm{dBm}]$ es el valor de RSSI obtenido entre un emisor y receptor.

\section{Algoritmos de triangulación}

La triangulación puede realizarse siguiendo dos métodos relevantes que describen desde el armado del sistema de ecuaciones hasta obtener la posición del móvil usando triangulación. Si bien los resultados son similares, es importante describir ambos métodos.

Según Nasca y Cojocariu, luego de obtener las mediciones entre señales y distancia, ocurre el problema de multilateración

$$
\left\{\begin{array}{c}
\left(x_{1}-x\right)^{2}+\left(y_{1}-y\right)^{2}=r_{1}^{2} \\
\left(x_{2}-x\right)^{2}+\left(y_{2}-y\right)^{2}=r_{2}^{2} \\
\vdots \\
\left(x_{n}-x\right)^{2}+\left(y_{n}-y\right)^{2}=r_{n}^{2}
\end{array}\right.
$$

Este problema se describe como sucesivas ecuaciones equivalentes a la cantidad de AP, que "ven" el dispositivo y pueden leer el valor del RSSI, $D=(x, y)$ es la posición del dispositivo, $\mathrm{AP}_{i}=\left(x_{i}, y_{i}\right)$ es la posición del $\mathrm{AP}_{i}$ y $r_{i}$ es la distancia medida desde el $\mathrm{AP}_{\mathrm{i}}$ al dispositivo. El sistema no es lineal pero es posible linealizar restando la $i$-ésima ecuación en las demás $n-1$.

$$
\left\{\begin{array}{l}
x_{1}^{2}-x_{n}^{2}-2\left(x_{1}-x_{n}\right) x+y_{1}^{2}-y_{n}^{2}-2\left(y_{1}-y_{n}\right) y=r_{1}^{2}-r_{n}^{2} \\
x_{2}^{2}-x_{n}^{2}-2\left(x_{2}-x_{n}\right) x+y_{2}^{2}-y_{n}^{2}-2\left(y_{2}-y_{n}\right) y=r_{2}^{2}-r_{n}^{2} \\
\vdots \\
x_{n-1}^{2}-x_{n}^{2}-2\left(x_{n-1}-x_{n}\right) x+y_{n-2}^{2}-y_{n}^{2}-2\left(y_{n-1}-y_{n}\right) y=r_{n-1}^{2}-r_{n}^{2}
\end{array}\right.
$$

En el caso ideal, todos los círculos se intersectan en un sólo punto, sin embargo, las medidas reales se ven afectadas por el error y los círculos se intersectan en más de un punto.

Nasca y Cojocariu infieren la localización espacial usando el método de eliminación Gaussiana en el sistema de ecuaciones propuesto.

Zhu y Feng, asume que hay $n$ APs y el nodo terminal o móvil se representa como $(x, y) . d_{i}$ representa a la distancia estimada entre el móvil y el $i$-ésimo nodo $\operatorname{AP}\left(x_{i}, y_{i}\right)$.

La diferencia entre la distancia real y la distancia estimada es expresada como $\rho_{\mathrm{i}}=\left|d_{i}-\mathrm{d}_{\mathrm{i}}^{\prime}\right|$. Asumiendo la existencia de un error , $\rho_{i}$, (distinto de cero), la solución propuesta para lograr la mejor estimación de localización es usar el algoritmo de mínimo cuadrados para hacer $\sum_{i=1}^{n} \rho_{i}^{2}$ mínima

A partir de la definición del sistema de ecuaciones que se puede obtener de un sistema de localización indoor, se obtiene la siguiente ecuación:

$$
A x=b
$$

Usualmente $d_{i}$ en b es desconocida, pero $d_{i}$ ' compuesta de $b$ ' puede ser estimada por el modelo mencionado anteriormente, entonces $\min \left(\sum_{i=1}^{n} \rho_{i}^{2}\right) \quad$ siginifica $\min \left|A X-b^{\prime}\right|$, luego la resolución de $\mathrm{x}$ ' es la siguiente:

$$
x^{\prime}=\left(A^{T} A\right)^{-1} A^{T} b^{\prime}
$$

A mayor cantidad de nodos AP disponibles, mayor es la precisión lograda pero con un mayor costo computacional y monetario. En casos reales, 3 nodos AP son suficientes para localizar un nodo desconocido, entonces consideramos $n=3$.

La elección de un método u otro para la localización espacial depende de la ubicación de los AP o el nodo terminal a localizar. Si se encuentran ubicados en los límites del ambiente indoor o en lugares donde la señal es débil, la localización espacial varía utilizando un método o el otro. Si la ubicación espacial no varía usando ambos métodos, entonces se está en presencia de un caso de localización exacta. La utilización de ambos métodos nos permite verificar la precisión en la localización. 


\section{Redes neuronales artificiales $(R N A)$}

Las redes neuronales artificiales son un paradigma de aprendizaje y procesamiento automático que se modelan matemáticamente para un problema en concreto y posteriormente formulan una solución mediante un algoritmo codificado que tenga una serie de propiedades que permitan resolver dicho problema. En el contexto de localización, los trabajos presentados por [17], [18] destacan la capacidad de las RNA de adaptarse a modelos no lineales.

El trabajo presentado en [19] presenta una solución basada en redes Perceptrón multicapa (MLP). El trabajo aborda la estimación de posición con el objetivo de optimizar la potencia de transmisión de nodos móviles y, así, reducir el consumo de los mismos. Así mismo, en [20], los autores proponen resolver el problema de localización mediante RSSI, sin considerar la pérdida de camino, pero aplicando filtros sobre la señal original. Esta información, en conjunto con la posición de los nodos, se integra dentro de una red neuronal multicapa, asegurando suficiente precisión para la aplicación indoor. Si bien los trabajos [18] y [20] están basados en la utilización de redes previamente entrenadas, [18] presenta un algoritmo adaptativo basado en la extracción de características de la señal para adaptar progresivamente los pesos de la red neuronal de manera que cuando se haya logrado la precisión deseada, la red deja de entrenarse.

Los trabajos mencionados consideran propiedades y características de la señal como información que alimenta a la red neuronal. Por ejemplo, algunas variantes de las RNA, muestran como entradas a las RSSI de cada uno de los AP, y las salidas son las probabilidades de estar en cada una de las localizaciones(Fig. 1).

Este método tarda mucho tiempo en entrenarse y necesita un conjunto de datos robustos, en algunos casos requiere de un pre procesamiento de los datos crudos, y presenta el problema de overfitting. Además, aunque la fase de entrenamiento se haya realizado correctamente, no se garantiza que ante los datos reales la respuesta sea la adecuada. Sin embargo, parece de los métodos más prometedores para estimar la localización del móvil en el posicionamiento en interiores.

En este trabajo se propone un entrenamiento y posterior estimación de una red cuyas entradas consideran la salidas de los algoritmos [14], [15] y [16]. El entrenamiento se realiza considerando las salidas de los tres algoritmos y la posición relativa real del nodo con respecto a los APs. Luego del proceso de entrenamiento, las redes neuronales poseen los pesos adecuados y cuando algún dispositivo móvil se encuentre en el área cubierta por esos nodos, se podrá obtener la posición del dispositivo en función a la distancia que reciban los nodos y los pesos adquiridos por la red en la etapa de entrenamiento.

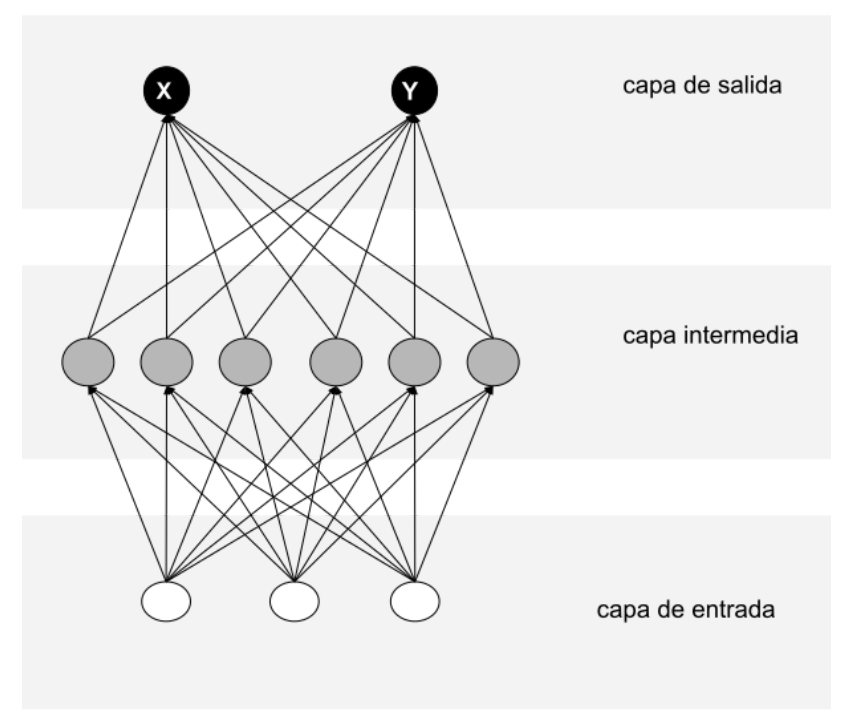

Fig. 1. Perceptrón multicapa con una capa intermedia [4].

\section{Desarrollo EXPerimental}

\section{A. Incremento de precisión de estimación de distancia}

Se realizaron ensayos experimentales utilizando dos placas de prototipos nodeMCU que proveen una ganancia de salida de $+25 \mathrm{dBm}[15,16]$ separados a diferentes distancias, uno utilizado como estación (terminal) y el otro como AP. La distancia se calculó en función de cada algoritmo. Para los tres ensayos se consideró el mismo tiempo de muestreo. Se realizaron mediciones y estimaciones de los tres algoritmos a distancias reales de $0.5,1,1.5,2,3$, y 4 metros. Los algoritmos de estimación fueron contrastados mediante el indicador de Error Porcentual Absoluto Medio (MAPE) para cada una de las distancias y los resultados se presentan en la Fig.2.

La Fig. 2 demuestra que si bien los métodos poseen un cierto grado de eficiencia, sus indicadores de error son elevados. En otro aspecto, se deduce que la utilización de un método en particular no es efectiva, sino que puede dependiendo de la distancia, es conveniente utilizar un método en particular.. Se puede observar que a una distancia igual a $1.5 \mathrm{~m}$ el MAPE es elevado, se debe a que ciertos algoritmos de estimación de distancia presenta una zona ciega producto de la señal recibida (RSSI), ya que la señal recibida no es lineal con la posición del móvil.

De forma experimental, se observó que a cortas distancias el algoritmo que mejor se adapta es Zhu y Feng, mientras que en distancias mayores, presenta un mejor desempeño el algoritmo de Barai, Bisbal y Sau. Si bien el valor MAPE del enfoque propuesto por Barai, Biswal y Sau es ligeramente superior al de Zhu y Feng, éste se mantiene constante a diferentes distancias, lo que muestra una ventaja del algoritmo. En otro aspecto, se destaca el promedio de error casi constante que posee el algoritmo de Nasca y Cojocariu, pero su uso puede ser descartado debido a que el índice de error promedia el $50 \%$. 


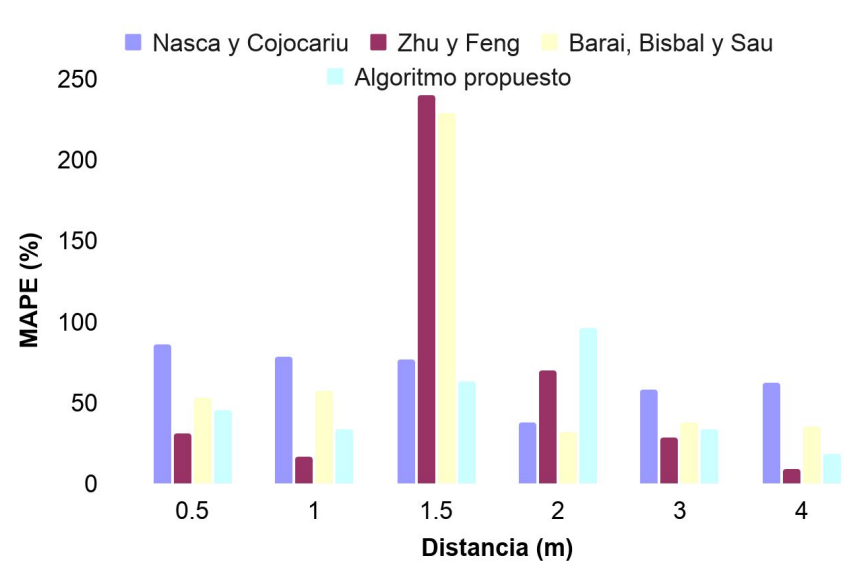

Fig. 2. Valores MAPE obtenidos en base a las distancias experimentales para los algoritmos seleccionados.

Considerando los resultados obtenidos se plantea una solución que combina los resultados de los algoritmos seleccionados a fin de lograr un aumento en la precisión. Se implementó una red neuronal del tipo perceptron (MLP) con tres neuronas de entrada y una neurona de salida. Presenta una tasa de aprendizaje de 0.7 y un momentum de $0.8 \mathrm{La}$ red neuronal fue entrenada a partir de los datos obtenidos de los métodos seleccionados considerando la distancia real como parámetro de entrada de entrenamiento para la estimación de la distancia. El error de entrenamiento fue del 0.024 y el porcentaje de validación de los resultados fue de 38.4. La configuración del MLP es experimental y seguramente se puede optimizar. Se evaluaron los indicadores de predicción de error cuadrático medio (MSE), error cuadrático medio (RMSE), error absoluto medio (MAE), tanto para la solución propuesta como los métodos escogidos. Los resultados de los indicadores se presentan en la Tabla I.

Los resultados obtenidos demuestran que el uso de una red neuronal entrenada a partir de las estimaciones de los métodos permite reducir los índices de error en un factor promedio del $25 \%$. Por otra parte, los índices de error absolutos y cuadráticos también se ven reducidos. Se realizaron pruebas, utilizando redes neuronales del tipo perceptrón multicapa, sin embargo los resultados de precisión de estas arquitecturas resultaron inferiores.

\section{B. Incremento del posicionamiento de localización espacial}

Para realizar la ubicación espacial se utilizaron 4 nodeMCU, donde 3 de ellos se comportan como AP y 1 en modo Terminal, denominado $\mathrm{M}$. El punto $\mathrm{ZF}$ muestra la localización espacial usando el método de Zhu Feng, mientras que el punto $N C$ hace referencia a la localización espacial usando el método de Nasca y Cojocariu. En el trabajo de Barai Biswal y Sau no se presenta una solución para la localización espacial ya que su trabajo se basa en presentar un algoritmo optimizado para la estimación de distancia. La Fig. 3 presenta un mapa descriptivo del escenario planteado.

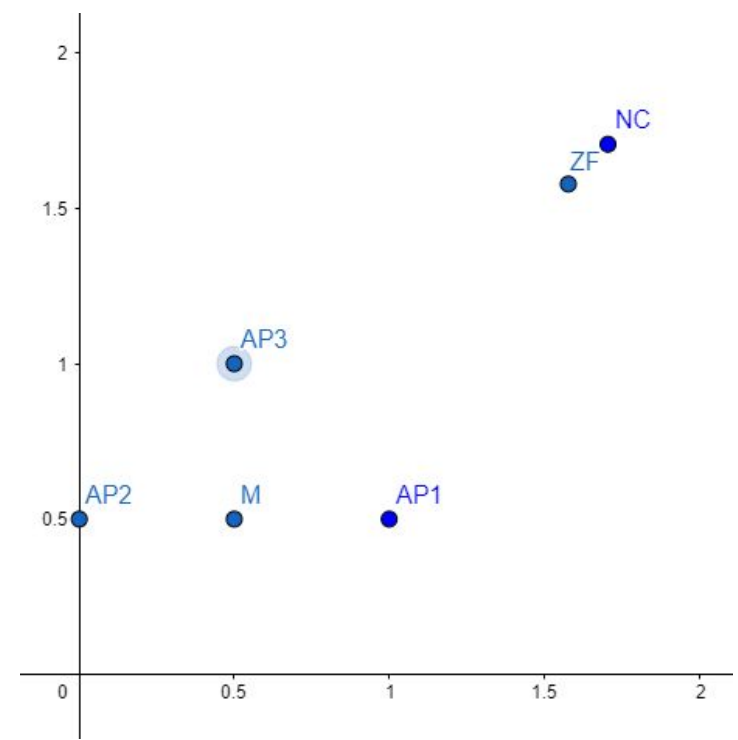

Fig. 3. Representación en ejes cartesianos del primer experimento realizado. Se muestra la ubicación de los AP y el terminal.

TABLA I

Indicadores de Error de los Métodos de Estimación de Posicionamiento INDOOR

\begin{tabular}{|l|c|c|c|}
\hline \multicolumn{1}{|c|}{ Algorítmo } & MAPE(\%) & RMSE (m) & MAE (m) \\
\hline Nasca y Cojocariu & 65.67 & 1.440 & 1.206 \\
\hline Zhu y Feng & 65.68 & 1.831 & 1.078 \\
\hline Barai, Biswal y Sau & 74.19 & 1.968 & 1.238 \\
\hline MLP & 40.49 & 1.066 & 0.723 \\
\hline
\end{tabular}

El nodo terminal $M$ escanea las redes AP cercanas y calcula su estimación de distancia sin usar la red neuronal con cada una de ellas. Estos resultados se expresan de la siguiente manera:

$$
\begin{aligned}
& A P 1 \Rightarrow(x-1)^{2}+(y-0.5)^{2}=0.274^{2} \\
& A P 2 \Rightarrow(x-0)^{2}+(y-0.5)^{2}=0.59178^{2} \\
& A P 3 \Rightarrow(x-0.5)^{2}+(y-1)^{2}=0.1704^{2}
\end{aligned}
$$

Usando cualquiera de los dos algoritmos de triangulación, se llega a un sistema de ecuacion lineal de la forma $A x=b$

$$
\left[\begin{array}{ll}
2 & 0 \\
1 & 1
\end{array}\right]\left[\begin{array}{l}
x \\
y
\end{array}\right]=\left[\begin{array}{l}
1.3052 \\
1.3512
\end{array}\right]
$$

Los resultados usando tanto eliminación Gaussiana como la Ecuación 7 son $\mathrm{x}=0.6526 \mathrm{y}=0.6986$. Se puede observar cierto error mínimo al querer localizar un móvil usando estimación de distancia sin usar la red neuronal.

Con el objetivo de utilizar una red neuronal que permita fusionar el resultado de los diversos algoritmos, resulta necesario el entrenamiento previo del modelo. La información de entrada proporcionada para el entrenamiento corresponde a los valores de los distintos algoritmos de estimación de distancia mientras que la salida a ser ajustada se realiza considerando la distancia real medida. Una vez entrenada la red neuronal, se obtienen un conjunto de pesos relacionados con la ponderación que posee cada algoritmo para poder predecir distancias. 


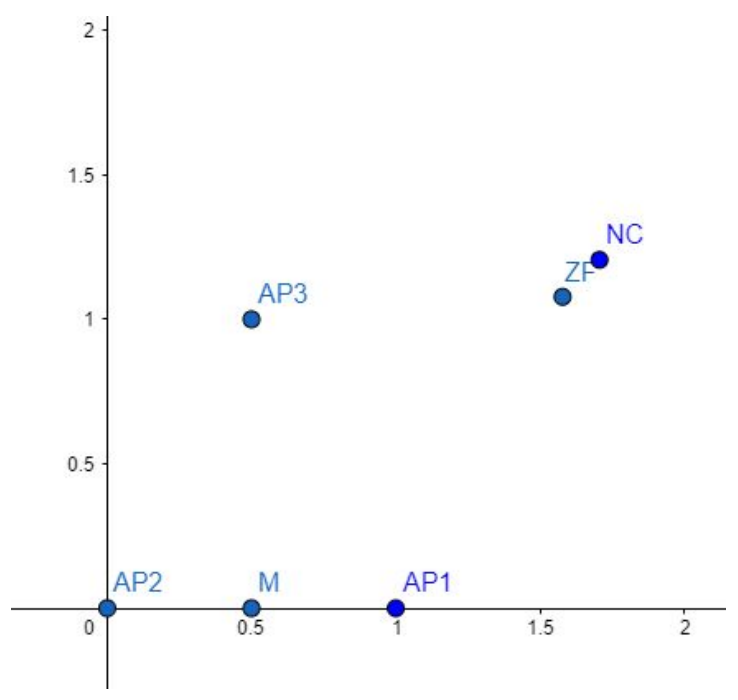

Fig. 4. Representación en ejes cartesianos del segundo experimento realizado. Se muestra la ubicación de los AP y el terminal

Utilizando la red neuronal para la estimación de distancia, se propone el siguiente sistema de ecuaciones:

$$
\begin{aligned}
& A P 1 \Rightarrow(x-1)^{2}+(y-0.5)^{2}=0.7624^{2} \\
& A P 2 \Rightarrow(x-0)^{2}+(y-0.5)^{2}=0.8145^{2} \\
& A P 3 \Rightarrow(x-0.5)^{2}+(y-1)^{2}=0.7462^{2}
\end{aligned}
$$

Los resultados de posición fueron $x=0.5411 y=0.5655$. Se puede observar que la estimación de la posición mejoró, siendo el resultado casi exacto.

Otro escenario donde se realizaron mediciones, se presenta en la Fig. 4

Se puede observar que tanto los AP1 y AP2 como el nodo móvil, se trasladan al límite inferior, es decir al eje de las abscisas.

Los datos obtenidos junto a la estimación de distancia, si usar la red neuronal son los siguiente:

$$
\begin{aligned}
& A P 1 \Rightarrow(x-1)^{2}+(y-0)^{2}=0.4917^{2} \\
& A P 2 \Rightarrow(x-0)^{2}+(y-0)^{2}=0.7837^{2} \\
& A P 3 \Rightarrow(x-0.5)^{2}+(y-1)^{2}=0.1962^{2}
\end{aligned}
$$

El sistema de ecuaciones, $A x=b$, se forma de la siguiente manera:

$$
\left[\begin{array}{cc}
1 & -2 \\
-1 & -2
\end{array}\right]\left[\begin{array}{l}
x \\
y
\end{array}\right]=\left[\begin{array}{c}
-0.1224 \\
1.8224
\end{array}\right]
$$

Los resultados obtenidos usando eliminación Gaussiana, son $x=1.5808$ e $y=0.8516$. En este caso la posición, usando la Ecuación 10, mostrando un $x=0$ e $y=0.9112$.

Luego, dentro del mismo escenario pero considerando las salida de la red neuronal entrenada se observa:

$$
\begin{aligned}
& A P 1 \Rightarrow(x-1)^{2}+(y-0)^{2}=0.7951^{2} \\
& A P 2 \Rightarrow(x-0)^{2}+(y-0)^{2}=0.8628^{2} \\
& A P 3 \Rightarrow(x-0.5)^{2}+(y-1)^{2}=0.7543^{2}
\end{aligned}
$$

El sistema de ecuaciones, $A x=b$, se forma de la siguiente manera:

$$
\left[\begin{array}{cr}
1 & -2 \\
-1 & -2
\end{array}\right]\left[\begin{array}{l}
x \\
y
\end{array}\right]=\left[\begin{array}{c}
-0.1377 \\
1.4255
\end{array}\right]
$$

Resolviendo este sistema de ecuaciones mediante eliminación Gaussiana, la localización obtenida es $(0.6439$, 0.3908). Usando la Ecuación 7, el resultado del posicionamiento es $(0.7816,0.3219)$, haciendo referencia el primer valor al eje $x$ y el segundo valor al eje $y$. Esta referencia es válida para ambas posiciones descritas.

Se puede observar en este experimento que la posición no es exacta, sin embargo se demuestra que la localización mejora usando red neuronal del tipo perceptrón en la estimación de distancia. Si bien, en los límites del mapa presenta problemas para localizar. Este es un problema vigente en los sistemas de posicionamiento indoor.

\section{CONCLUSIÓN}

El presente trabajo describe la relación entre los algoritmos que utilizan las fórmulas de pérdida de espacio libre con los algoritmos que relacionan la media y el desvío estándar. Se realizó la implementación de tres algoritmos de estimación de distancia previamente publicados y fueron comparados cuantitativamente respecto al error. Los resultados indicaron que no existe una superioridad en cuanto a la precisión de uno respecto al resto, y que además sus índices de error son elevados para mediciones precisas. Sin embargo, al fusionar los resultados de los métodos en una red neuronal, el error absoluto medio pudo ser reducido en un $25 \%$, lo que representa una mejora significativa.

Con respecto a la localización espacial, se plantearon dos métodos para realizar la triangulación: utilizando ecuaciones derivadas de conceptos del álgebra matricial, y utilizando eliminación Gaussiana.. Ambos métodos permiten resolver un sistema de ecuación lineal. Se comprobó que el uso de la red neuronal disminuye el error de localización. Si bien, la localización espacial presenta errores en los límites del mapa, el error continúa siendo mucho menor con respecto a si se utilizan sólo los métodos seleccionados.

Se plantea continuar el desarrollo mediante el análisis de otros métodos de localización de bajo costo computacional, que puedan ser incorporados como entradas a la red neuronal, así como también explotar el entrenamiento de la red para incrementar su precisión. Se propone además extender el trabajo, no solo a la medición de la distancia entre dos nodos, sino a la ubicación espacial del nodo en diferentes escenarios para permitir una localización generalización con menor error de precisión.

El desarrollo propuesto se integrará junto a un sistema de recolección y análisis de datos biométricos para ser utilizado como soporte a la seguridad de operarios.

\section{Agradecimientos}

Este trabajo fue parcialmente financiado por la SeCAT de la Universidad Nacional del Centro de la Provincia de Buenos Aires, Argentina (Código de Proyecto 03/C287).

\section{REFERENCIAS}

[1] C. Lago Gonzales, J. C. Sepulveda Peña, R. Barroso Abrea, F. Oscar Fernandez Peña, F. Macia Paz, J. Lorenzo, "Sistema para la generación automática de mapas de rendimiento. Aplicación en la agricultura de precisión”. Vol 29, n¹, pp 59-69. IDESIA, Chile Enero- Abril 2011. 
[2] F. Jimenez Moral,. "ShoppingBeacon: Sistema asistencial en superficies comerciales basado en posicionamiento en interiores". Trabajo fin de grado. Universidad de Castilla-La mancha. Escuela Superior de Informática. Septiembre, 2015.

[3] F. Dwiyasa, M.-H. Lim, "A survey of problems and approaches in ecuacfigurawireless-based indoor positioning", Proc. Int. Conf Indoor Positioning Indoor Navigat., pp. 1-7, Oct. 2016.

[4] L. Diaz, A. Tabernilla, F. Perez Costoya, "Sistema de Localización de Interiores". Trabajo fin de carrera. Universidad politécnica de madrid. Facultad de informática.

[5] Eva M. Garcia Polo. "Técnicas de Localización en Redes Inalámbricas de Sensores”. Instituto de informática de Albanecete. Departamento de Sistemas Informáticos. Universidad de Castilla-La Mancha

[6] Shahra, E. Q., Sheltami, T. R., \& Shakshuki, E. M. (2020). A comparative study of range-free and range-based localization protocols for wireless sensor network: Using cooja simulator. In Sensor Technology: Concepts, Methodologies, Tools, and Applications (pp. 1522-1537). IGI Global.

[7] Xiao, J., Liu, Z., Yang, Y., Liu, D., \& Han, X. (2011, June). Comparison and analysis of indoor wireless positioning techniques. In 2011 International conference on computer science and service system (CSSS) (pp. 293-296). IEEE

[8] Mekelleche, F., \& Haffaf, H. Classification and comparison of range-based localization techniques in wireless sensor networks. Journal of Communications, 12(4), 221-227, 2017.

[9] A. Tahat, G. Kaddoum, S. Yousefi, S. Valaee, and F. Gagnon, “A look at the recent wireless positioning techniques with a focus on algorithms for moving receivers," IEEE Access, vol. 4, pp. 6652-6680, 2017.

[10] N. H. Nguyen and K. Dogançay, "Optimal geometry analysis for multistatic TOA localization,'” IEEE Trans. Signal Process., vol. 64, no. 16, pp. 4180-4193, Aug. 2016.
[11] G. Wang, A. M.-C. So, and Y. Li, "Robust convex approximation methods for TDOA-based localization under NLOS conditions,' IEEE Trans. Signal Process., vol. 64, no. 13, pp. 3281-3296, Jul. 2016.

[12] L. Liu and H. Liu, "Joint estimation of DOA and TDOA of multiple reflections in mobile communications," IEEE Access, vol. 4, pp. 3815-3823, 2016

[13] Q. Luo, Y. Peng, J. Li, and X. Peng, "RSSI-based localization through uncertain data mapping for wireless sensor networks," IEEE Sensors J., vol. 16, no. 9, pp. 3155-3162, May 2016.

[14] Xiuyan Zhu, Yuan Feng. "RSSI-based Algorithm for Indoor Localization". College of Information Science and Engineering, Ocean University of China, Qingdao, China

[15] Survankar Barai, Debajyoti Biswal y Buddhadeb Sau, "Estimate Distance Measurement using NodeMCU ESP8266 based on RSSI technique". Departament on Mathematics, jadavpur University, Kolkata - 700032, India, pp 170-171

[16] I. Nasca, T. Cojocariu, Indoor Positioning System Using Wi-Fi Signal. Today Software Magazine 26. November 2015

[17] H. Chen, Y. Zhang, W. Li, X. Tao, and P. Zhang, "ConFi: Convolutional neural networks based indoor Wi-Fi localization using channel state information," IEEE Access, vol. 5, pp. 18 066-18 074, Sep. 2017.

[18] S.-H. Fang and T.-N. Lin, "Indoor location system based on discriminant-adaptive neural network in IEEE 802.11 environments," IEEE Transactions on Neural Networks, pp. 1973-1978, Nov 2008.

[19] erns," in: Proceedings of AINS2002, UCLA, pp. 1-13, 2002. [19] X. Lu, H. Zou, H. Zhou, L. Xie, and G.-B. Huang, "Robust extreme learning machine with its application to indoor positioning," IEEE Transaction on Cybernetics, vol. 46, no. 1, pp. 194-205, Jan. 2016.

[20] H. Dai, W. hao Ying, and J. Xu, "Multi-layer neural network for received signal strength-based indoor localization," IET Communications, vol. 10, pp. 717-723, Jan. 2016. 